\title{
Oscillatory behavior of coercivity in $\mathrm{Bi} / \mathrm{Co}$ bilayers
}

\author{
Jen-Hwa Hsu ${ }^{\mathrm{a}, *}$, Zhi-Long Xue ${ }^{\mathrm{a}}$, Ta-Chieh Huang ${ }^{\mathrm{a}}$, Zung-Hang Wei ${ }^{\mathrm{b}}$, Mei-Feng Lai ${ }^{\mathrm{c}}$ \\ a Department of Physics, National Taiwan University, Taipei 106, Taiwan \\ ${ }^{\mathrm{b}}$ Department of Power Mechanical Engineering, National Tsing-Hua University, Hsinchu 300, Taiwan \\ ${ }^{\mathrm{c}}$ Department of Engineering Science and Ocean Engineering, National Taiwan University, Taipei 106, Taiwan
}

Available online 20 November 2006

\begin{abstract}
The $\mathrm{Bi} / \mathrm{Co}$ bilayers on the glass substrate with various thicknesses of Bi underlayer were prepared by DC sputtering method. A remarkable enhancement of the coercivity $H_{\mathrm{c}}$ of sputtered thin Co films was observed. Besides, its coercivity at room temperature was found to oscillate as a function of Bi thickness with a period of about $22 \mathrm{~nm}$. The range of oscillation was observed at least up to $80 \mathrm{~nm}$. The origin of the oscillation in the coercivity of cobalt film was thought to arise from the specific 3D growing mechanism of bismuth layer.

(C) 2006 Elsevier B.V. All rights reserved.
\end{abstract}

PACS: 75.70.-i; 74.25.Ha; 81.05.Bx; 68.35.Ct

Keywords: Magnetic film and multilayer; Magnetic property; Semimetal; Interface structure and roughness

Ferromagnetic bilayer with a non-magnetic underlayer was intensely studied in recent years driven by the intriguing physics such as magnetic anisotropy, exchange bias and the variation of coercivity. As is well known, the magnetic properties of bilayer thin films are strongly influenced by microstructure such as interface roughness and crystallinity. In the past, the studies were limited to the metal underlayers [1] and semiconductor underlayers [2]. In this investigation, the semi-metallic $\mathrm{Bi}$ was chosen as the underlayer material. The low melting point of $\mathrm{Bi}$ enables the layer structures to be easily modified and to enable the variation in the magnetic behaviors with interfacial structure to be observed. A large enhancement and oscillatory behavior in the coercivity $H_{\mathrm{c}}$ of sputtered thin Co films with a Bi underlayer was found.

The Bi/Co bilayers were prepared by the DC sputtering method. The 7059 corning glass was used as the substrate. Herein, the thickness of Co layer was kept at $6 \mathrm{~nm}$, while the thickness $t$ of $\mathrm{Bi}$ underlayer was varied from 1 to $100 \mathrm{~nm}$.

\footnotetext{
${ }^{*}$ Corresponding author. Tel.: + 886233665162 ; fax: +886233665892.

E-mail address: jhhsu@phys.ntu.edu.tw (J.-H. Hsu).
}

The crystalline structure was investigated by the X-ray diffraction (XRD) using $\mathrm{CuK}_{\alpha}$ radiation. The surface morphology was examined by the atomic force microscopy (AFM). The magnetic loops were obtained from the vibrating sample magnetometer (VSM).

The XRD results indicated that only the rhombohedral phase of bismuth was present. No diffraction peaks corresponding to Co were found due to larger scattering factor of Bi. Besides, no additional phase other than Bi has been detected. Fig. 1 displayed the AFM images for the Bi/ Co bilayers. The AFM measurements revealed that large clusters with the size of about $330 \mathrm{~nm}$ were randomly distributed as shown in Fig. 1(a). The clusters seem to connect with each other. In contrast as shown in Fig. 1(b), the small granules have higher density and tend to be aligned. Besides, deep narrow valleys are observed.

The representative $M-H$ loops at room temperature were plotted in Fig. 2. It is noted that the shape of the loops does not change with the thickness of Bi but is significantly different from that of single Co layer. Besides, the width of the loop varies with $t$, but it does not increase steadily. For example, the loop for $t=18 \mathrm{~nm}$ was narrower than those for both $t=4 \mathrm{~nm}$ and $t=27 \mathrm{~nm}$. 
The coercivity of glass $/ \mathrm{Bi}(t) / \mathrm{Co}$ bilayers at room temperature was found to oscillates as a function of $\mathrm{Bi}$ thickness with a period of about $22 \mathrm{~nm}$ and the range of
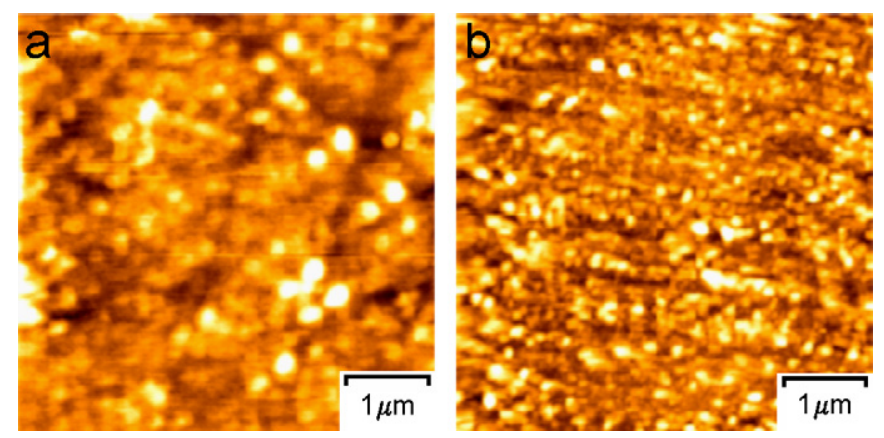

Fig. 1. Representative AFM micrographs of the surface of glass $/ \mathrm{Bi}(t) / \mathrm{Co}$ films: (a) $t=18 \mathrm{~nm}$ and (b) $t=27 \mathrm{~nm}$,

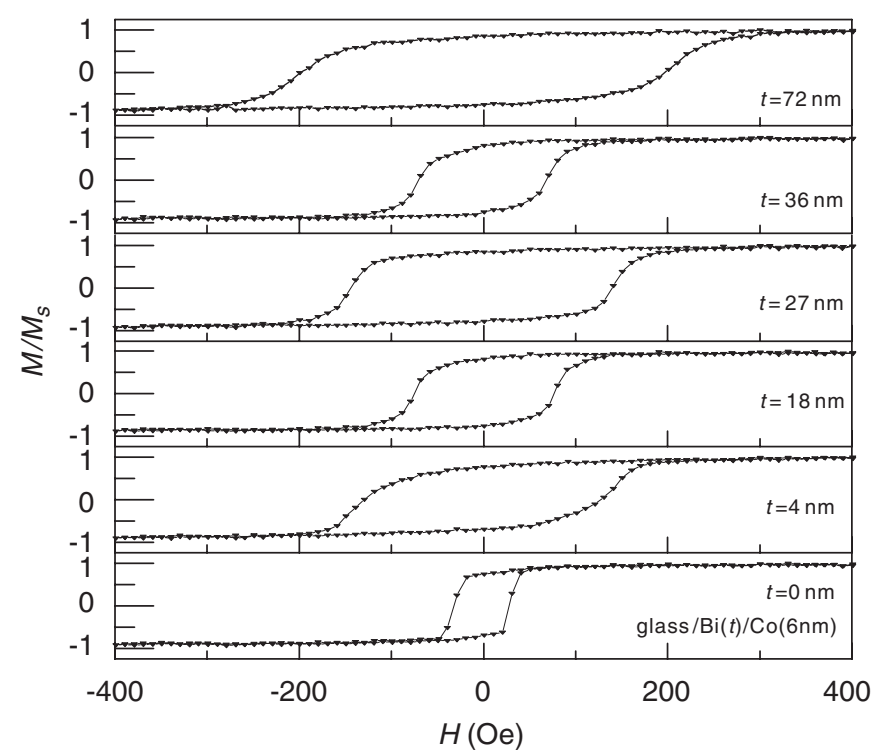

Fig. 2. Representative magnetization loops for the samples with applied field along the sample plane.

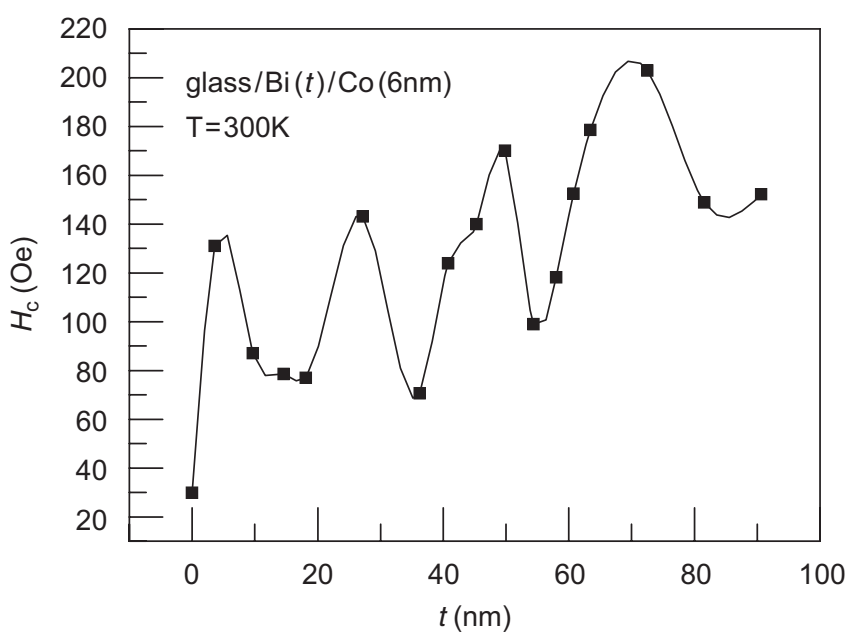

Fig. 3. Coercivity as a function of increasing Bi underlayer thickness. oscillation was extended at least up to $80 \mathrm{~nm}$ as shown in Fig. 3. This type of oscillation was not detected in glass/ $\mathrm{Ag}(t) / \mathrm{Co}$ bilayer system [3]. The small $H_{\mathrm{c}}$ is associated with a smoother surface while the high $H_{\mathrm{c}}$ is found for the rougher films as shown in Figs. 1(a) and (b). The origin of the oscillation in the coercivity of cobalt film was thought to come from the growing mechanism of bismuth. And the oscillation in $H_{\mathrm{c}}$ of Co films is not caused by the coupling effect because the oscillation in $H_{\mathrm{c}}$ disappears if the $\mathrm{Bi}$ film is deposited on the Co film instead. According to Stranski-Krastanov model [4] for epitaxy, islands form during film deposition in addition to layer-by-layer accumulation. By means of AFM observation, the Bi surface was found to be significantly rough made of many steep islands. The schematic pictures were given in Fig. 4 to explain the possible epitaxy process of the Bi film. Steep islands form in the course of deposition, and narrow, deep valleys develop between these islands as shown in Fig. 4(a). However, the heights of these islands are impossible to unlimitedly increase. When the islands become high enough, the higher islands collapse and the valleys are filled with collapsing atoms at the same time. Wider and smoother valleys form as shown in Fig. 4(b). As continuing on the growth process, once again the islands gradually grow higher, steeper, and the valleys become narrower as shown in Fig. 4(c). The whole process continues. Therefore, in the course of the $\mathrm{Bi}$ layer deposition, the aspect ratio (vertical length)/(lateral length) of the interface roughness varies with $\mathrm{Bi}$ thickness

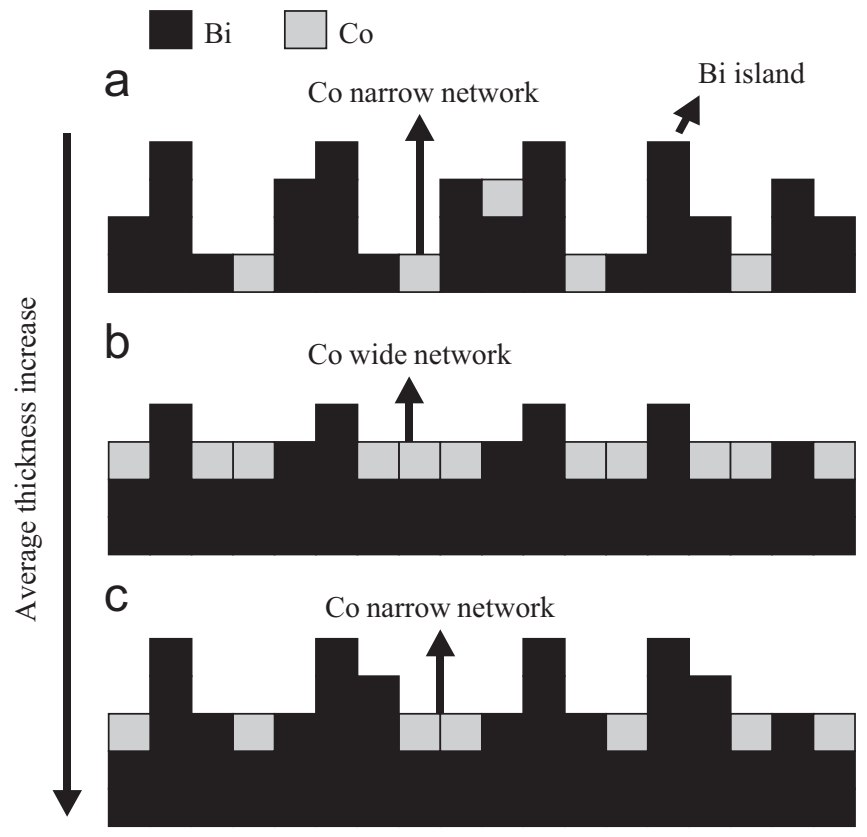

Fig. 4. Epitaxy process of bismuth and cobalt films. (a) steep islands form in the course of epitaxy, and narrow network of cobalt develops surrounding the islands, (b) smooth islands form, and wide network of cobalt develops surrounding the islands, and (c) steep islands form, and narrow network of cobalt develops surrounding the islands. 
periodically. After the completion of $\mathrm{Bi}$ film growth, the Co film of $6 \mathrm{~nm}$ thickness was deposited thereon. Co network structure forms in the valleys surrounding the $\mathrm{Bi}$ islands. In Fig. 4(a), the width of the Co network is very small because the $\mathrm{Bi}$ valleys are very narrow and steep. However in Fig. 4(b) wider Co networks have formed because of the smoother $\mathrm{Bi}$ valleys. Along with the periodically high and low islands in $\mathrm{Bi}$ deposition process, the width of the Co network forming in the $\mathrm{Bi}$ valleys also exhibites the periodic variation. The coercivity of a narrower network is higher than that of a wider network [5], so the oscillation in the coercivity of Co film with Bi thickness is expected. Furthermore, the uniaxial magnetic anisotropy was found for the films with high $H_{\mathrm{c}}$.

In summary the oscillation behavior in $H_{\mathrm{c}}$ was observed in glass/Bi/Co bilyer system, which was believed due to the periodic variation of interface structure.
This work was supported by the National Science Council of Taiwan (Contract Nos. NSC94-2112-M-002039 and NSC94-2120-M-002-011).

\section{References}

[1] H.S. Jung, W.D. Doyle, J.E. Wittig, J.F. Al-Sharab, J. Bentley, Appl. Phys. Lett. 81 (2002) 2415.

[2] J.J. Krebs, B.T. Jonker, G.A. Prinz, J. Appl. Phys. 61 (1987) 2596; Y. Chye, V. Huard, M.E. White, P.M. Petroff, J. Appl. Lett. 80 (2002) 449.

[3] J. Swerts, K. Temst, N. Vandamme, C. Van Haesendonck, J. Magn. Magn. Mater. 240 (2002) 380.

[4] I.N. Stranski, L. von Krastanov, Akad. Wiss. Lit. Mainz Abh. Math. Naturwiss. K1. 146 (1939) 797.

[5] I. Ruiz-Feal, L. Lopez-Diaz, A. Hirohata, J. Rothman, C.M. Guertler, J.A.C. Bland, L.M. Garcia, J.M. Torres, J. Bartolome, F. Bartolomé, M. Natali, D. Decanini, J. Chen, J. Magn. Magn. Mater. 242-245 (2002) 597. 